\title{
Mountain Waves Produced by a Stratified Boundary Layer Flow. Part I: Hydrostatic Case
}

\author{
FRANÇOIS LotT, BRuno DEREMBLE, AND ClÉMENT SOUFFLET \\ Laboratoire de Météorologie Dynamique, PSL Research Institute, Ecole Normale Supérieure, Paris, France
}

(Manuscript received 19 September 2019, in final form 30 January 2020)

\begin{abstract}
A hydrostatic theory for mountain waves with a boundary layer of constant eddy viscosity is presented. It predicts that dissipation impacts the dynamics over an inner layer whose depth is controlled by the innerlayer scale $\delta$ of viscous critical-level theory. The theory applies when the mountain height is smaller or near $\delta$ and is validated with a fully nonlinear model. In this case the pressure drag and the wave Reynolds stress can be predicted by inviscid theory, if one takes for the incident wind its value around the inner-layer scale. In contrast with the inviscid theory and for small mountains the wave drag is compensated by an acceleration of the flow in the inner layer rather than of the solid earth. Still for small mountains and when stability increases, the emitted waves have smaller vertical scale and are more dissipated when traveling through the inner layer: a fraction of the wave drag is deposited around the top of the inner layer before reaching the outer regions. When the mountain height becomes comparable to the inner-layer scale, nonseparated upstream blocking and downslope winds develop. Theory and the model show that (i) the downslope winds penetrate well into the inner layer and (ii) upstream blocking and downslope winds are favored when the static stability is strong and (iii) are not associated with upper-level wave breaking.
\end{abstract}

\section{Introduction}

The impact of small- to medium-scale mountains on the atmospheric dynamics has been intensively studied over the last 50 years by two quite distinct communities. One community is studying how mountains modify the turbulent boundary layer (Jackson and Hunt 1975), an issue that is central in the context of wind resource modeling (Ayotte 2008) or dune formation (Charru et al. 2013). The associated theories form the basis of subgrid-scale orography parameterizations, where the enhancement of turbulence caused by mountains is modeled by increasing the terrain roughness length (Wood and Mason 1993). Wood et al. (2001) used fully nonlinear simulations to extend the theory and improve the estimate of the depth over which the mountain drag is deposited. These parameterizations are used for mountains with horizontal scales smaller than $5000 \mathrm{~m}$ (Beljaars et al. 2004). At these scales one can expect that the horizontal scale of the mountains $L$ is such that the advective time scale $L / u_{0}$ is smaller than the inverse of the buoyancy frequency $N^{-1}$. This ensures that the flow behaves according to neutral flow dynamics.

Corresponding author: François Lott, flott@lmd.ens.fr
The second community is more focused on mountain dynamical meteorology. It studies the onset of downslope winds, foehn, and trapped waves using theories and models where internal gravity waves control the dynamics, and where the boundary layer is often neglected. The relevance of the approach is illustrated by Sheridan et al. (2017), where a near-linear mountain wave model permits to interpret wind perturbations due to mountain wave events over the United Kingdom. The associated theory is extremely vast in itself (Durran 1990). Among other things, this theory has been used to predict realistic partitions between upper-level and lower-level wave drag and orographic blocking, which are concepts that are used in parameterizations of subgrid-scale orography with horizontal scales $L>$ $5000 \mathrm{~m}$ (Lott and Miller 1997). Note that this type of parameterization is still used in atmospheric models, and even in the models with horizontal resolution that resolve these scales (Sandu et al. 2015; Pithan et al. 2016). In fact, it is not so clear whether there is a critical mountain size $(L=5000 \mathrm{~m})$ below which the flow would only impact the boundary layer and above which the flow would only impact the waves. We actually believe that this criteria is quite ad hoc and should depend on the nature of the flow. 
Because boundary layer dynamics is highly controlled by the inviscid dynamics aloft, and because in mountain meteorology the wave forcing is embedded into the boundary layer, it soon appeared that the two communities should make some effort to integrate results from the other community. It is in this context that Hunt et al. (1988) and Belcher and Wood (1996) included stratification and gravity waves in boundary layer theories over mountain. Belcher and Wood (1996) showed that when the Froude number $F=u_{0} / N L$ is smaller than 1 , the mountain drag is due to mountain gravity waves (rather than boundary layer effect) and is well predicted by linear gravity mountain wave theory. This result actually depends on the height at which one chooses the reference velocity $u_{0}$ and reference Brunt-Väisälä frequency $N$. As we shall see, in the absence of background wind curvature, the relevant altitude to compute these quantities is that of the inner layer, which is the altitude where disturbance advection by the background wind is balanced by dissipation. Still in this context but in the mountain meteorology community, studies using numerical model show that the boundary layer drag reduces downslope windstorms and mountain waves (Richard et al. 1989; Ólafsson and Bougeault 1997). More recent observations show that the atmospheric boundary layer can absorb downward-propagating waves and weaken trapped lee waves (Smith et al. 2002; Jiang et al. 2006). These last results have motivated a series of theoretical studies on the interaction between the boundary layer and mountain waves. All so far use crude parameterizations of the boundary layer: Smith et al. (2006) uses a bulk boundary layer model, Lott (2007) used constant eddy viscosity, and Lott (2016) uses linear drags (Newtonian cooling and Rayleigh drag).

Despite these simplifications, these studies reproduce the increase in trapped waves absorption when stability increases, insisting on cases where the incident wind is weak near the ground. This near-surface critical-level situation, a situation that was little studied because it poses fundamental problems in the inviscid mountain wave theory, was nevertheless found to produce interesting dynamics. Near-surface critical level favors downslope windstorms and foehn (Lott 2016; Damiens et al. 2018) and permits to establish a bridge between trapped lee waves and Kelvin-Helmholtz instabilities (Lott 2016; Soufflet et al. 2019). Interestingly, the critical-level mechanism that is a priori a dissipative mechanism turned out to be extremely active dynamically.

To summarize, there are two descriptions of the interaction between boundary layers and mountain waves: on the one hand boundary layer studies tell that the pressure drag is controlled by the mountain wave dynamics outside of the boundary layer, but imposes very simplified dynamics outside of it (Belcher and Wood 1996). And on the other hand, "mountain wave" studies that give great attention to the potential impact of a boundary layer on mountain waves but that use very simplified boundary layer representation (Smith et al. 2006). We actually believe that there is still room to develop a theory where the boundary layer and the mountain wave field fully interact in a comprehensive way. We see at least three reasons for this. The first is that in mountain wave theory, the gravity wave (GW) field is controlled by the low-level flow amplitude, and it is not obvious to tell at which (or over which) altitude it should be measured in the absence of strong wind curvatures. Second, we know that the inviscid dynamics potentially produces downslope winds in the stratified case and it could be interesting to test if they extend down to the surface and well into the inner layer. Last, we know that the pressure drag is controlled by the wave drag in the stable case, but we do not know if a fraction of the wave drag could and should be deposited into the inner layer rather than being radiated away. This issue could have important consequences for the formulation of parameterizations.

The purpose of the present paper is to answer these questions in the reference case where the boundary layer is parameterized via a constant kinematic eddy viscosity $\nu$. This case has the unique merit that, while the Couette profile with constant shear $u_{0 z}$ is an exact solution, we can handle the interactions with topography using the stratified viscous solutions derived by Hazel (1967) and Baldwin and Roberts (1970). However, a consequence of using uniform wind shear is that the "boundary layer depth" of the incident flow is infinite, it is therefore totally distinct from the "inner-layer depth" over which the waves are affected by dissipation and that scales as

$$
\delta=\left(\frac{\nu L}{u_{0 z}}\right)^{1 / 3}
$$

These simplifications of uniform viscosity and background shear were made in the literature of the late 1950s by Benjamin (1959) and Smith (1973) in the context of flows over water waves and dunes, respectively. Since then, we are well aware that such a "laminar" approach is an extreme idealization. A reason is that boundary layer dynamics tends to produce winds with strong shear near the surface but that vary much more slowly at higher altitudes (the associated curvatures defining the "boundary layer depth" quite precisely). To defend our choice nevertheless, we can recall that in the 
atmosphere the low-level wind shears are not only due to the boundary layer: they are also related to the largescale dynamics. This has been shown for instance in experiments done by Sheridan et al. (2007) and Doyle et al. (2011), where they observe strong shears over few kilometers above the ground. This being said, we will have to keep in mind that models with constant eddy viscosity probably overstate the significance of the lowlevel shear stresses on the waves and pressure drag (Sykes 1978).

The plan of the paper is as follows. In section 2 we derive the theory in the hydrostatic case. In section 3 we discuss the pressure drag and wave momentum fluxes it predicts. In section 4 we analyze the onset of downslope winds. As our theory is linear except for the lower boundary condition, our results are checked against fully nonlinear simulations in section 5 . In section 6 , we discuss further the significance of works on boundary layer using constant eddy viscosity. We also discuss in this section how our results could be useful to understand the dynamics in more realistic cases. In appendix A, we detail some aspects of the theory, and in appendix B we provide details on the numerical implementation of the model.

\section{Theory}

\section{a. Basic equations}

We consider a background flow solution of the viscous equations,

$$
u_{0}(z)=u_{0 z} z, \quad \rho_{0}(z)=\rho_{r}+\rho_{0 z} z,
$$

where the wind shear $u_{0 z}$ and stratification $\rho_{0 z}$ are both constant, and that is incident on a Gaussian ridge of characteristic length $L$ and maximum height $H$ :

$$
h(x)=H e^{-x^{2} /\left(2 L^{2}\right)} .
$$

Following quite conventional approaches (Beljaars et al. 1987; Belcher and Wood 1996), we consider obstacles of small slope and use linear equations. To characterize the factors that control the dynamics we also normalize the response by introducing the "outer" scaling:

$$
\begin{aligned}
(x, z) & =L(\bar{x}, \bar{z}), \quad\left(u^{\prime}, w^{\prime}\right)=u_{0 z} L(\bar{u}, \bar{w}), \\
\left(p^{\prime}, b^{\prime}\right) & =\left(\rho_{r} u_{0 z}^{2} L^{2} \bar{p}, u_{0 z}^{2} L \bar{b}\right),
\end{aligned}
$$

where $u^{\prime}$ and $w^{\prime}$ are the horizontal and vertical wind disturbances whereas $b^{\prime}$ is the buoyancy disturbance. With this scaling, and making the conventional "Prandtl" approximation that the vertical derivatives dominate the viscous terms, the $2 \mathrm{D}$ Boussinesq hydrostatic linear equations write

$$
\begin{aligned}
\bar{z} \partial_{\bar{x}} \bar{u}+\bar{w} & =-\partial_{\bar{x}} \bar{p}+\bar{\nu} \partial_{\bar{z}}^{2} \bar{u}, \\
0 & =-\partial_{\bar{z}} \bar{p}+\bar{b}, \\
\bar{z} \partial_{\bar{x}} \bar{b}+J \bar{w} & =P^{-1} \bar{\nu} \partial_{\bar{z}}^{2} \bar{b}, \\
\partial_{\bar{x}} \bar{u}+\partial_{\bar{z}} \bar{w} & =0
\end{aligned}
$$

with no-slip boundary conditions:

$$
\begin{aligned}
\bar{h}(\bar{x})+\bar{u}(\bar{x}, \bar{h}) & =0, \quad \bar{w}(\bar{x}, \bar{h})=0, \quad \text { and } \\
J \bar{h}(\bar{x})+\bar{b}(\bar{x}, \bar{h}) & =0 \quad \text { at } \quad \bar{h}=S e^{-\bar{x}^{2} / 2} .
\end{aligned}
$$

In Eqs. (5) and (6),

$$
J=-\frac{g \rho_{0 z}}{\rho_{r} u_{0 z}^{2}}, \quad P=\frac{\nu}{\kappa}, \quad S=\frac{H}{L}, \quad \text { and } \quad \bar{\nu}=\frac{\nu}{u_{0 z} L^{2}}
$$

are a Richardson number, a Prandtl number, a slope parameter, and an inverse Reynolds number, respectively.

To help establish where the waves are produced and where they are dissipated, we next derive from Eqs. (5) a wave-action budget. As this is not often done in mountain waves literature, we recall that the interest is to define a quantity $A$ that is quadratic (to measure the wave amplitude locally) and conservative in the adiabatic frictionless case. For action we chose the pseudomomentum, because its vertical flux $F^{z}$ is closely related to the mountain wave Reynolds stress ${ }^{1}$ [see further discussions in Durran (1995) and Lott (1998)]. Although the exact form of the wave action is rigorously derived when starting from Hamiltonian dynamics (Scinocca and Shepherd 1992), we can directly use the formula for the pseudomomentum $A$ derived in this paper, and derive a budget that includes dissipation by doing the formal operation:

$$
\frac{\bar{b}}{J} \partial_{\bar{z}}[\text { Eq. }(5 \mathrm{a})]+\frac{\bar{u}_{\bar{z}}}{J}[\text { Eq. (5c)]. }
$$

\section{After few integrations by parts one obtains}

\footnotetext{
${ }^{1}$ It is actually interesting to recall that the seminal paper on wave-mean flow interaction by Eliassen and Palm (1961) was about mountain waves.
} 


$$
\begin{aligned}
& \frac{\partial}{\partial \bar{x}} \underbrace{(\underbrace{\frac{\partial_{\bar{z}} \bar{u}}{J} \bar{b}}_{A}+\frac{\bar{b}^{2}}{2 J}+\frac{\bar{u}^{2}}{2})}_{F^{x}}+\frac{\partial}{\partial \bar{z}} \underbrace{\bar{u} \bar{w}}_{F^{z}} \\
& =\underbrace{\bar{\nu} \frac{\bar{b}}{\bar{J}} \overline{\partial_{\bar{z}}^{2}} \partial_{\bar{z}} \bar{u}+P^{-1} \bar{\nu} \partial_{\bar{z}} \bar{u} \overline{\partial_{\bar{z}}^{2}} \bar{b}}_{Q},
\end{aligned}
$$

where $F^{x}$ and $F^{z}$ the horizontal and vertical components of the pseudomomentum flux, and where $Q$ is the production/destruction of action by dissipative processes. Note that $F^{x}$ includes the horizontal advection of action by the background flow $\bar{z} A$. As we search inflow solutions that are linear, we next express them in terms of Fourier transform,

$$
\begin{aligned}
& \bar{w}(\bar{x}, \bar{z})=\int_{-\infty}^{+\infty} \overline{\mathbf{w}}(\bar{k}, \bar{z}) e^{i \bar{k} \bar{x}} d \bar{k}, \quad \text { where } \\
& \overline{\mathbf{w}}(\bar{k}, \bar{z})=\frac{1}{2 \pi} \int_{-\infty}^{+\infty} \bar{w}(\bar{x}, \bar{z}) e^{-i \bar{k} \bar{x}} d \bar{x},
\end{aligned}
$$

which transforms Eqs. (5) into

$$
\begin{aligned}
i \bar{k} \bar{z} \overline{\mathbf{u}}+\overline{\mathbf{w}} & =-i \bar{k} \overline{\mathbf{p}}+\bar{\nu} \partial_{\bar{z}}^{2} \overline{\mathbf{u}}, \\
i \bar{k} \bar{z} \overline{\mathbf{b}}+J \overline{\mathbf{w}} & =P^{-1} \bar{\nu} \partial_{\bar{z}}^{2} \overline{\mathbf{b}}, \\
\overline{\mathbf{b}} & =\partial_{\bar{z}} \overline{\mathbf{p}}, \\
i \bar{k} \overline{\mathbf{u}}+\partial_{\bar{z}} \overline{\mathbf{w}} & =0 .
\end{aligned}
$$

\section{b. Solutions}

For high Reynolds number $\bar{\nu} \ll 1$, the dynamics is inviscid at leading order. Each harmonics satisfy Eqs. (11) with $\bar{\nu}=0$, which results in $\overline{\mathbf{w}}$ satisfying

$$
\overline{\mathbf{w}}_{\bar{z} \bar{z}}+\frac{J}{\bar{z}^{2}} \overline{\mathbf{w}}=0 .
$$

Such equation has two solutions (Booker and Bretherton 1967):

$$
\bar{z}^{1 / 2 \pm i \mu}, \text { where } \mu=\sqrt{J-\frac{1}{4}} .
$$

When $\bar{k}>0$ and $J>0.25$, only the solution

$$
\overline{\mathbf{w}}_{I}(\bar{k}, \bar{z})=\bar{z}^{1 / 2+i \mu}
$$

corresponds to a gravity wave propagating upward. The cases with $\bar{k}<0$ are treated by complex conjugation and will not be discussed further. The cases with $J<0.25$ are degenerated in the hydrostatic approximation because the direction of vertical propagation cannot be used to distinguish between the two solutions in Eq. (13). This difficulty, which forbids to treat the weakly stratified cases (i.e., here when $J<1 / 4$ ), will be resolved in a future nonhydrostatic treatment of the inviscid solution.

To solve the inner layer we introduce the scaling,

$$
\begin{aligned}
\bar{z} & =\bar{\delta} \tilde{z}, \quad(\overline{\mathbf{u}}, \overline{\mathbf{w}})=(\tilde{\mathbf{u}}, \bar{\delta} \bar{k} \tilde{\mathbf{w}}), \\
(\bar{p}, \bar{b}) & =(\bar{\delta} \tilde{p}, \tilde{b}) \quad \text { where } \quad \bar{\delta}=\left(\frac{\bar{\nu}}{\bar{k}}\right)^{1 / 3} .
\end{aligned}
$$

At leading order, it transforms the full set of nondimensional Eqs. (5) into the sixth-order set:

$$
\begin{gathered}
\partial_{\tilde{z}}^{2} \tilde{\mathbf{u}}=i \tilde{z} \tilde{\mathbf{u}}+\tilde{\mathbf{w}}+i \tilde{\mathbf{p}}, \\
\partial_{\tilde{z}}^{2} \tilde{\mathbf{b}}=P(i \tilde{z} \tilde{\mathbf{b}}+J \tilde{\mathbf{w}}), \\
\partial_{\tilde{z}} \tilde{\mathbf{w}}=-i \tilde{\mathbf{u}}, \quad \partial_{\tilde{z}} \tilde{\mathbf{p}}=\tilde{\mathbf{b}} .
\end{gathered}
$$

This set of equations can be reduced to one single equation for $\tilde{\mathbf{w}}$,

$$
\left(\partial_{\tilde{z}}^{2}-i P \tilde{z}\right)\left(\partial_{\tilde{z}}^{2}-i \tilde{z}\right) \partial_{\tilde{z}}^{2} \tilde{\mathbf{w}}=J P \tilde{\mathbf{w}},
$$

which has six independent solutions. Hazel (1967) and Baldwin and Roberts (1970) have found their asymptotic form when $\tilde{z} \rightarrow \infty$. Two grow exponentially as $\tilde{z} \rightarrow \infty$ and cannot be used (Van Duin and Kelder 1986), the four that remain have asymptotic forms:

$$
\begin{aligned}
& \tilde{\mathbf{w}}_{1} \underset{\tilde{z} \rightarrow \infty}{\approx} \tilde{z}^{1 / 2-i \mu}, \quad \tilde{\mathbf{w}}_{2} \underset{\tilde{z} \rightarrow \infty}{\approx} \tilde{z}^{1 / 2+i \mu}, \quad \tilde{\mathbf{w}}_{3} \underset{\tilde{z} \rightarrow \infty}{\approx} \tilde{z}^{-5 / 4} e^{-(2 \sqrt{i} / 3) z^{3 / 2}}, \\
& \tilde{\mathbf{w}}_{4} \underset{z \rightarrow \infty}{\approx} \tilde{z}^{-9 / 4} e^{-(2 \sqrt{i P} / 3) \tilde{z}^{3 / 2}} .
\end{aligned}
$$

In Lott (2007), these four solutions are evaluated over the entire domain $0<\tilde{z}<\infty$, that is, by using the asymptotic forms (18) above $\tilde{z}=5$ and integrating down Eq. (17) from $\tilde{z}=5$ to $\tilde{z}=0$ with a Runge-Kutta algorithm. We will essentially proceed like this here (some serious convergence issues are discussed in appendix A). We then notice that the inner function $\tilde{\mathbf{w}}_{2}$ matches the upward inviscid solution (14) and that $\tilde{\mathbf{w}}_{3}$ and $\tilde{\mathbf{w}}_{4}$ decay exponentially fast with altitude, which permit to tell that all the combinations of $\tilde{\mathbf{w}}_{2}, \tilde{\mathbf{w}}_{3}$, and $\tilde{\mathbf{w}}_{4}$ are uniform solutions that can match $\overline{\mathbf{w}}_{I}$. We therefore search a uniform approximation of the vertical velocity under the form

$$
\begin{aligned}
\overline{\mathbf{w}}(\bar{k}, \bar{z})= & \bar{k} \bar{\delta}(k)\left\{f _ { 2 } ( \overline { k } ) \tilde { \mathbf { w } } _ { 2 } \left[\begin{array}{c}
\bar{z} \\
\bar{\delta}(\bar{k})
\end{array}+f_{3}(\bar{k}) \tilde{\mathbf{w}}_{3}\left[\frac{\bar{z}}{\bar{\delta}(\bar{k})}\right]\right.\right. \\
& \left.+f_{4}(\bar{k}) \tilde{\mathbf{w}}_{4}\left[\begin{array}{c}
\bar{z} \\
\bar{\delta}(\bar{k})
\end{array}\right]\right\},
\end{aligned}
$$



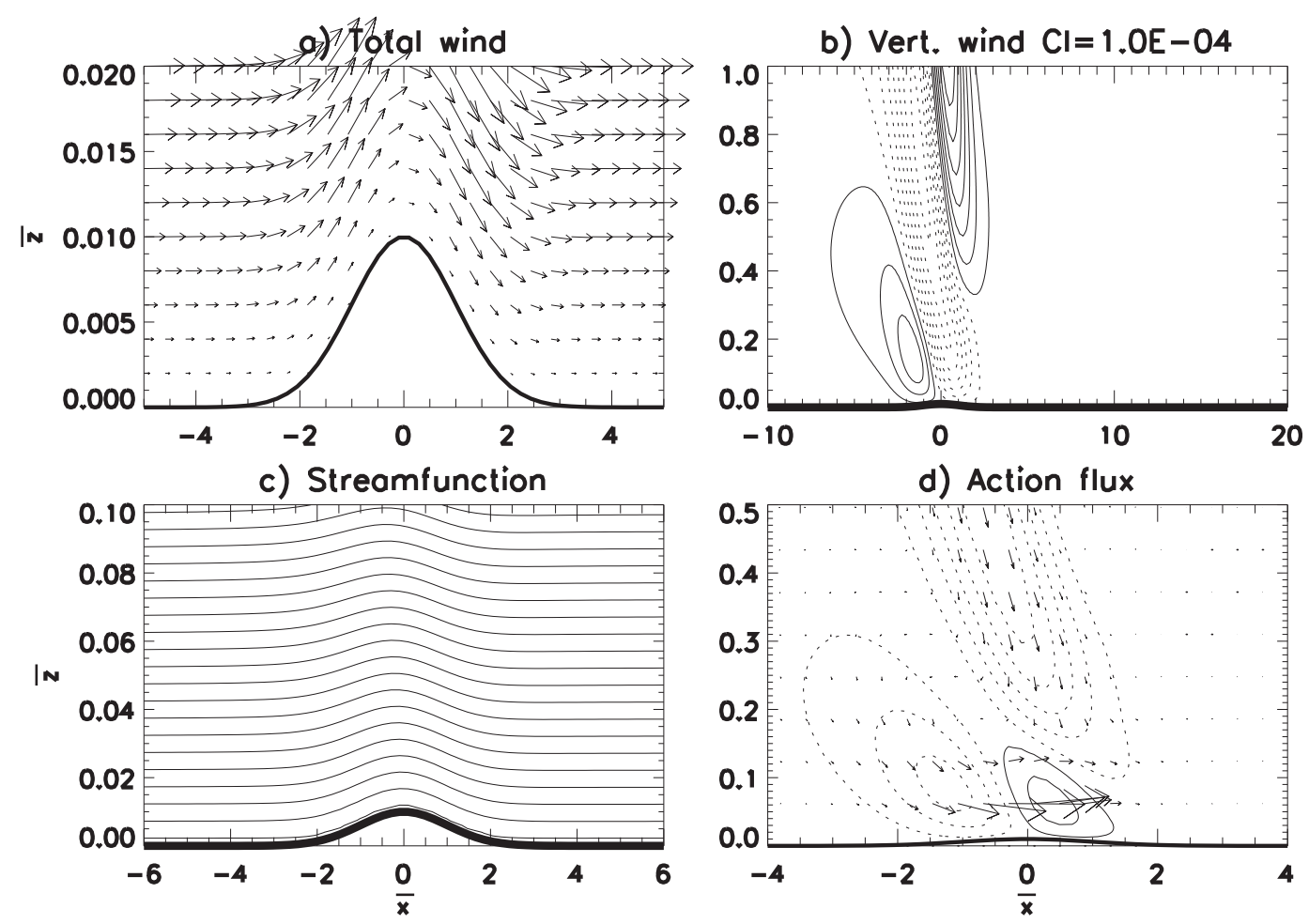

FIG. 1. Physical fields predicted by the theory in the hydrostatic case and when $J=4, S=0.01, \bar{\delta}=0.1$. (a) Total wind vector $(\bar{z}+\bar{u}, \bar{w})$. (b) Vertical wind $\bar{w}$. (c) Total streamfunction $\bar{\psi}$ defined by $\partial_{\bar{z}} \overline{\boldsymbol{\psi}}=\bar{z}+\bar{u}$. (d) Vertical flux of action $F^{z}$ (contours) and action flux vector $\left(F^{x}, F^{z}\right)$. In (b) and (d) the negative values are dashed.

where all fields are expressed using outer variables, and with similar expression for $\overline{\mathbf{u}}$ and $\overline{\mathbf{b}}$ deduced from Eq. (11). To evaluate the unknown functions $f_{2}, f_{3}$, and $f_{4}$, we write the boundary conditions:

$$
\begin{aligned}
\bar{w}(\bar{x}, \bar{h}) \approx & \int_{-\infty}^{+\infty} \bar{k} \bar{\delta}(\bar{k})\left[f_{2}(\bar{k}) \tilde{\mathbf{w}}_{2}(\tilde{h})+f_{3}(\bar{k}) \tilde{\mathbf{w}}_{3}(\tilde{h})\right. \\
& \left.+f_{4}(\bar{k}) \tilde{\mathbf{w}}_{4}(\tilde{h})\right] e^{i \bar{k} \bar{x}} d \bar{k}=0 \\
\bar{u}(\bar{x}, \bar{h}) \approx & \int_{-\infty}^{+\infty}\left[f_{2}(\bar{k}) \tilde{\mathbf{u}}_{2}(\tilde{h})+f_{3}(\bar{k}) \tilde{\mathbf{u}}_{3}(\tilde{h})\right. \\
& \left.+f_{4}(\bar{k}) \tilde{\mathbf{u}}_{4}(\tilde{h})\right] e^{i \bar{k} \bar{x}} d \bar{k}=-\bar{h}(\bar{x}) \\
\bar{b}(\bar{x}, \bar{h}) \approx & \int_{-\infty}^{+\infty}\left[f_{2}(\bar{k}) \tilde{\mathbf{b}}_{2}(\tilde{h})+f_{3}(\bar{k}) \tilde{\mathbf{b}}_{3}(\tilde{h})\right. \\
& \left.+f_{4}(\bar{k}) \tilde{\mathbf{b}}_{4}(\tilde{h})\right] e^{i \bar{k} \bar{x}} d \bar{k}=-J \bar{h}(\bar{x})
\end{aligned}
$$

where $\tilde{h}(\bar{x}, \bar{k})=\bar{h}(\bar{x}) / \bar{\delta}(\bar{k})$. Once discretized in the horizontal and spectral domain, the set of Eqs. (20) corresponds to three linear equations for $f_{2}(\bar{k}), f_{3}(\bar{k})$, and $f_{4}(\bar{k})$ that can be inverted with conventional matrix inversion routines (see appendix B for more details on the numerical treatment).

\section{Mountain wave fields and drags}

We plot in Fig. 1 the flow response when the inverse Reynolds number $\bar{\nu}=0.001$, the slope parameter $S=$ 0.01 , the Richardson number $J=4$, and the Prandtl number $\operatorname{Pr}=0.5$. This last parameter will stay unchanged in the rest of the paper: we have found moderate sensitivity of the upper wave fields to this parameter as long as its value stays around 1 . In this setup, the characteristic inner-layer scale is that of the dominant harmonic $\bar{k}=1$, that is, $\bar{\delta}(\bar{k}=1)=\bar{\nu}^{1 / 3}=0.1$, which is also the nondimensional form of the inner-layer scale in Eq. (1). The inner-layer scale is therefore much larger than the mountain slope.

The total wind at low level in Fig. 1a contours well the obstacle and the vertical velocity field (Fig. 1b) highlight a system of well-defined upward-propagating gravity waves. We notice that the streamfunction in Fig. 1c follows well the orography, up to at least the inner-layer scale $\bar{\delta}(1)=0.1$. For each altitudes below and around $\bar{\delta}(1)$ the streamlines are displaced vertically over distances that are near the mountain height $S$, and 
the vertical velocity when $\bar{z} \approx \bar{\delta}$ should scale as $\overline{\mathbf{w}} \approx[\bar{\delta}(1) / 2] S$ to follow the streamlines. We therefore propose that the wave amplitude corresponds to the inviscid case when a uniform flow of amplitude $\bar{\delta}(1) / 2$ (the average of the incident wind over the inner-layer scale) is incident over a mountain of maximum height $S$. Far aloft and in the sheared case, the vertical velocity should therefore scale as $\overline{\mathbf{w}}=0\{[\sqrt{\bar{z} \bar{\delta}(1)} / 2] S\}$, where the square root corresponds to the $1 / 2$ factor in the exponent of the inviscid solution [Eq. (14)]. This qualitative argument tells that the amplitude of $\bar{w}$ should be around $S \bar{\delta}(1) / 2=5 \times 10^{-4}$ at $\bar{z}=1$, which is in qualitative agreement with what is found in Fig. $1 b$.

We follow this line of qualitative reasoning and propose as predictor of the wave momentum flux and mountain pressure drag,

$\overline{\bar{u}} \bar{w}(\bar{z})=\int_{-\infty}^{+\infty} \bar{u}(\bar{x}, \bar{z}) \bar{w}(\bar{x}, \bar{z}) d \bar{x}, \quad \operatorname{Dr}=-\int_{-\infty}^{+\infty} \bar{p}(\bar{x}, \bar{h}) \frac{\partial \bar{h}}{\partial \bar{x}} d \bar{x}$,

the inviscid linear hydrostatic pressure drag produced by a uniform wind of intensity $\bar{\delta}(1) / 2$ incident on the orography given by $\bar{h}(\bar{x})$ in Eq. (6), and which exact value is

$$
-\frac{\bar{\delta}(1)}{2} \sqrt{J} S^{2}=-\mathrm{Dr}_{\mathrm{GWP}}
$$

Henceforth, we will refer to $\operatorname{Dr}_{\mathrm{GWP}}$ as the gravity wave drag amplitude predictor. Figure 2 shows that this predictor is a good estimate for the drag given by the theoretical model for a very large range of $J$ and slope $S$. We conclude that the pressure drag is well controlled by the inviscid GW dynamics outside of the inner layer, the GWs being forced by the undulations of the inner layer produced by the mountain. This picture where the inner layer forces the (inviscid) dynamics aloft, and that the pressure drag is ultimately controlled by this inviscid dynamics follows the general principle of boundary layer theories that pressure is approximately constant across the inner layer.

This predictor of the surface pressure drag is nevertheless misleading if we take it as a measure of the effect of the mountain on the large-scale flow, as is generally done in mountain meteorology. The reason is that, in a steady state, our waves are forced indirectly by the distortion of the inner layer produced by the mountain rather than directly by the mountain as in the inviscid case. To establish this, we return to Fig. 1d where we plotted the waves pseudomomentum flux vector. Aloft the inner layer this flux clearly points down, as expected for mountain GWs propagating upward (Durran 1995;

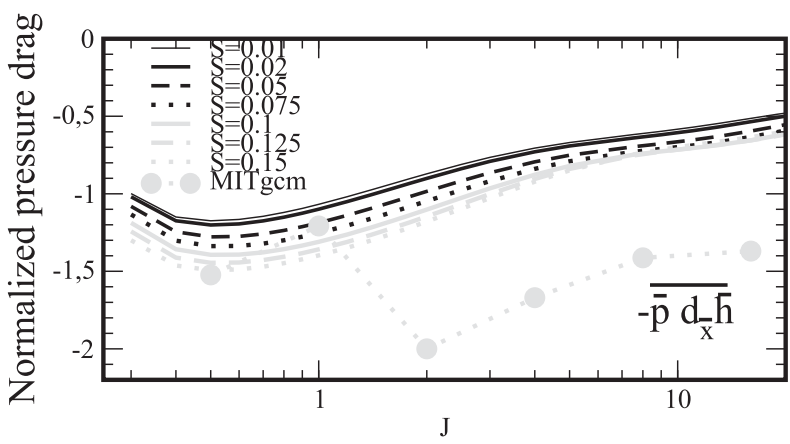

FIG. 2. Surface pressure drag predicted by theory and normalized by the amplitude of the inviscid linear mountain gravity wave drag produced by a mountain of height $H$ in a uniform flow of intensity $U=u_{0}\left[\delta\left(L^{-1}\right) / 2\right]$ and of stratification $N: U N H^{2}$ in nondimensional form: $[\bar{\delta}(1) / 2] \sqrt{(J)} S^{2}$; see Eq. (22)\}. Gray dots are from the MITgcm with $S=0.15$.

Lott 1998), but within the inner layer, it points very strongly from the upstream sector toward the downstream sector. This is to be contrasted with the inviscid case where this flux goes through the surface and produces an exchange of momentum between the fluid and the solid ground.

This result suggests that the acceleration that balances the gravity wave drag is not communicated to Earth's surface but rather to the inner layer. This last statement is confirmed in Fig. 3a, where we plot the wave stress as a function of altitude. The wave stress is null at the surface, increases with altitude before reaching a constant value at altitudes above $\bar{z}>5 \bar{\delta}(1)$ typically. As is often the case for viscous boundary layers, the depth over which dissipation is significant seems to be around 5 times the inner-layer scale $\bar{\delta}(1)$, so we will systematically make the distinction between the inner-layer scale $\bar{\delta}(1)$ and the inner-layer depth [around $5 \bar{\delta}(1)]$. The flux emitted at the top of the inner layer [above $5 \bar{\delta}(1)$ ] is around half the pressure drag, at least when $J \approx 1$. Such value stays comparable to the theoretical drag but suggests that substantial wave dissipation occurs when the wave travels vertically through the inner layer [in our scenario where the waves are forced around $\bar{\delta}(1)]$. This erosion of the pressure drag toward the gravity wave stress is even more significant when $J$ increases. This is again consistent with a qualitative argument: for large values of $J$, the waves oscillate more rapidly in the vertical according to Eq. (14) and are more affected by viscous dissipation. This difficulty in converting the pressure drag into a momentum flux as stability increases makes that for $J>4$ typically, there is a minimum in $\overline{\bar{u} \bar{w}}$ in the middle of the inner layer [between $2 \bar{\delta}(1)$ and $5 \bar{\delta}(1)$ ]: part of the momentum given to the inner layer near the surface is restored back around the top of the inner layer. 
a) Reynolds stress

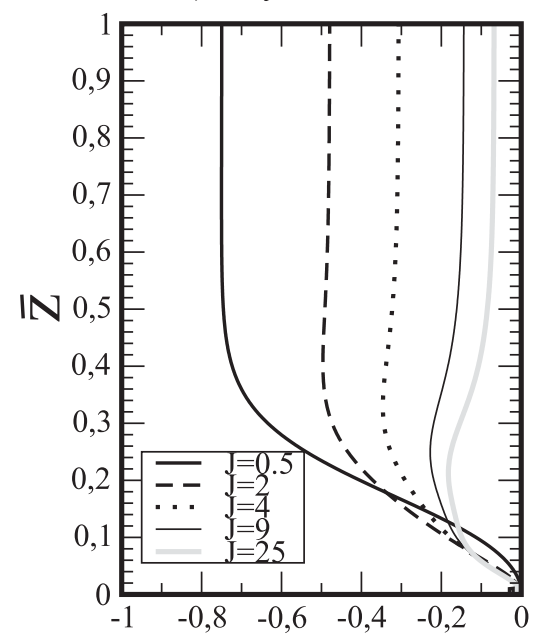

b) Pressure and Viscous stress

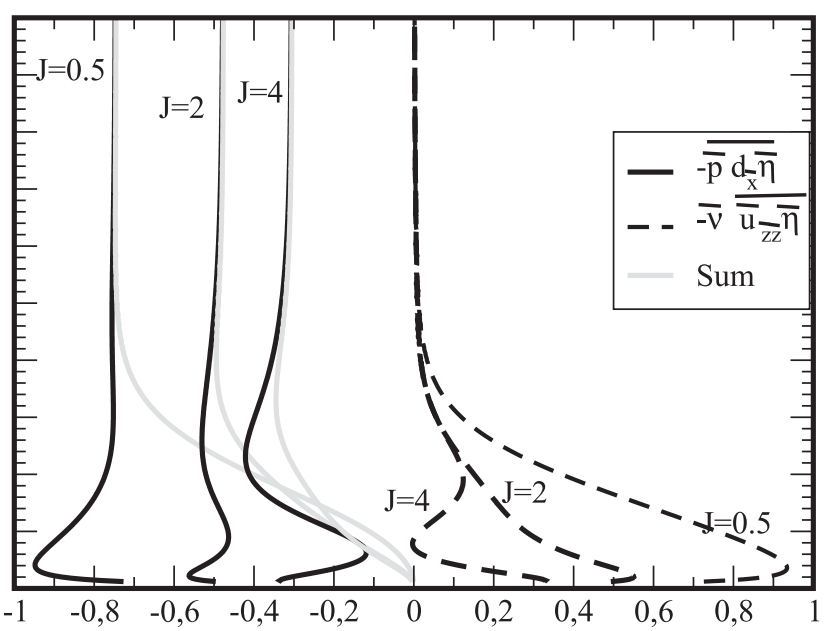

FiG. 3. (a) Vertical profiles of the normalized wave Reynolds stress $\overline{\bar{u}} \bar{w}$ for $S=0.01$. (b) Pressure (black solid) and viscous (black dashed) stresses as defined on the RHS of Eq. (23).

To understand what can replace the Reynolds stress to balance the pressure drag, it is important to return to the initial Eliassen and Palm's (1961) paper, where it is shown that the momentum flux is related to the pressure force exerted in the horizontal direction on an undulating surface. In the linear stationary case, this relation is obtained by multiplying a momentum equation [Eq. (5a) in our case] by the vertical displacement of streamlines $\bar{\eta}$ and after integration by part over $\bar{x}$ we get

$$
\overline{\bar{u}} \bar{w}=-\overline{\bar{p}} \partial_{\bar{x}} \bar{\eta}-\bar{\nu} \overline{\left(\bar{\eta} \partial_{\bar{z}}^{2} \bar{u}\right)} \text {, where } \bar{z} \partial_{\bar{x}} \bar{\eta}=\bar{w} .
$$

In the inviscid case the pressure stress equals the Reynolds stress, but this is no longer true in the viscous scenario where dissipation plays a nonnegligible role. To illustrate how dissipation becomes significant for small slopes, we plot the two terms on the right-hand side and their sum for three values of $J$ in Fig. 3b. After verification that the sum in Fig. $3 \mathrm{~b}$ exactly equals the Reynolds stresses in Fig. 3a, we see that Reynolds stress and the pressure drag only coincide well above the inner layer. Near the surface and in the lower part of the inner layer, the pressure drag is almost entirely balanced by the viscous drag.

This erosion of the pressure drag toward the wave Reynolds stress is summarized in Fig. 4a where we plot the Reynolds stress emitted in $\bar{z} \rightarrow \infty$ normalized by the predictor $\operatorname{Dr}_{\mathrm{GWP}}$. As already discussed, the emitted flux is half the predicted drag, but this result becomes sensitive to the stability $J$ : when $J$ is large, the emitted flux almost vanishes. This erosion of the pressure drag toward the Reynolds stress for large $J$ is less pronounced if we consider the minimum values in Fig. 4b. These minima are in general located in the middle of the inner layer [i.e., above $\bar{z}=\bar{\delta}(1)$ and below $5 \bar{\delta}(1)$, see Fig. 3a] such that for large $J$ some GW deceleration should be applied directly around the top of the inner layer [which we locate at $5 \bar{\delta}(1)]$.

\section{Nonseparated blocking and downslope winds}

To analyze what occurs in nonlinear situations we next consider cases where the slope $S$ becomes comparable to the inner-layer scale $\bar{\delta}(1)$. As a first example, the simulation in Fig. 5 corresponds to that in Fig. 1 but with
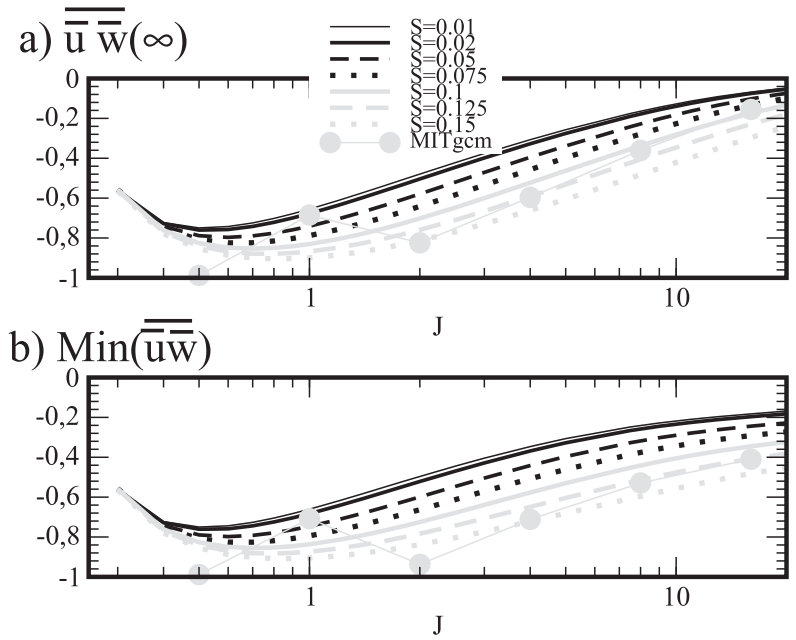

FIG. 4. (a) Normalized Reynolds stress emitted in the far field $\overline{\bar{u}} \bar{w}(\infty)$. (b) Minimum value of $\overline{\bar{u}} \bar{w}(z)$ for $0<\bar{z}<\infty$. Gray dots are from the MITgcm with $S=0.15$. 

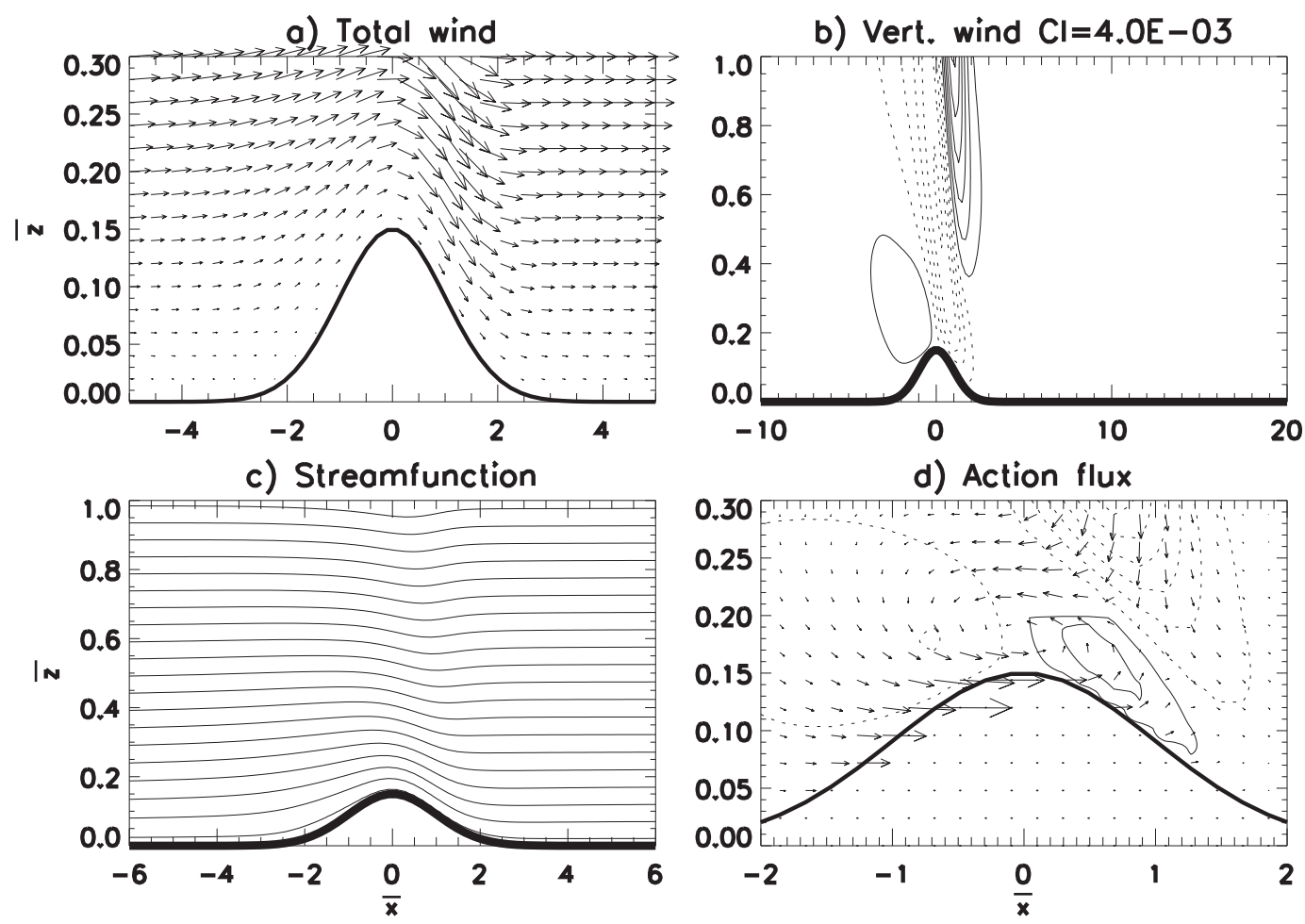

FIG. 5. As in Fig. 1, but for $S=0.15$.

$S=0.15$ instead of $S=0.01$. We readily notice that the total wind (Fig. 5a) presents an downslope-upslope asymmetry that is almost absent in Fig. 1a. The vertical velocity is around 30 times larger than in the small slope case, that is, 3 times larger than what should have been obtained if we applied a linear ratio of the slopes (Figs. 5b, 1b). The asymmetry in the winds is also visible in the streamfunction in Fig. 5c, with a pronounced descent on the lee side. Finally, the largest differences are maybe in the pseudomomentum flux vector in Fig. $5 \mathrm{~d}$. Now that the obstacle penetrates well into the inner layer, there is a substantial pseudomomentum flux across the surface. In opposition to the inviscid case (Lott 1998) we did not identify clear relations between this flux and the mountain drag, except that the total flux across the surface is on the same order of magnitude as the mountain drag when the slope approaches the innerlayer scale.

To appreciate more systematically the changes occurring when the slope parameter increases as a function of stability, we plot in Fig. 6 the vertical velocity fields for different values of $S$ and $J$. Figures $6 a-c$ are for a slope that is small compared to the inner-layer scale $[S=0.02<\bar{\delta}(1)]$ and Figs. $6 \mathrm{~d}-\mathrm{f}$ are for a slope that compares to it $[S=0.15 \approx \bar{\delta}(1)]$. The contour interval stays the same for all panels with a given slope, consistent with the fact that the kinematic boundary conditions are independent of $J$ [see Eq. (6)]. Between Figs. $6 \mathrm{a}-\mathrm{c}$ and $6 \mathrm{~d}-\mathrm{f}$ where the slope changes, the contour interval changes with a factor proportional to the slope ratio, that is, following a linear relation. For the small slope cases when $J$ increases (Figs. 6a-c), one sees that the wave amplitude in the far field decreases with $J$. If we recall that the vertical scale of variations of our solutions is inversely proportional to $J$, larger values of $J$ correspond to cases where the solutions oscillate more in the vertical direction, these plots are therefore consistent with the interpretation that with large $J$ the waves are more dissipated when they travel through the inner layer. When the slope increases, a second interesting behavior is worth noticing. When $J=1$ there are little differences between the patterns in Figs. $6 \mathrm{a}$ and $6 \mathrm{~d}$, which means that amplitudes varies linearly with $S$ (remember that the contour interval varies linearly with $S$ between the top and bottom rows). Again, we know since Lott (2016) and Damiens et al. (2018) that this is also related to the vertical scale of the waves: strong nonlinear effects enter the dynamics via the surface boundary condition and when the vertical wavelength at the top of the obstacle compares to the vertical wavenumber, a criteria that corresponds to $J>1$. As we see in Figs. 6e and 6f these nonlinear effects become substantial: for a given slope the wave amplitude now increases when $J$ increases. In addition to the enhanced 

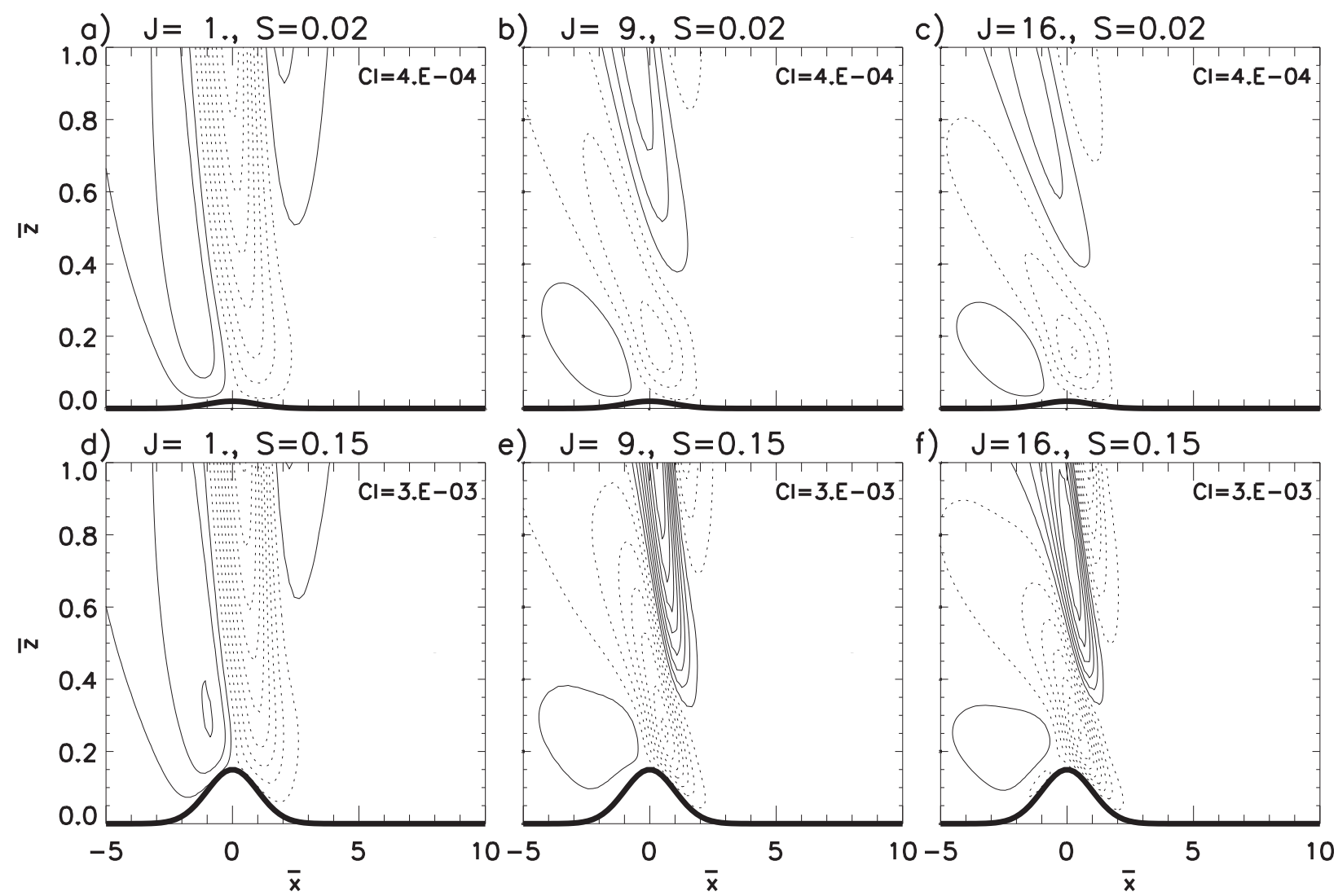

FIG. 6. Vertical velocity for different values of the Richardson number $J$ and of the slope $S$. Inner-layer depth $\delta(1)=0.1$. Contour intervals are shown in each panel.

emission due to nonlinearities, it is also plausible that the wave dissipation through the inner layer is less intense because the level of emission is located at a higher altitude than for smaller slopes. If we return to the emitted momentum fluxes in Fig. 4, a consequence of these enhanced emission and reduced dissipation when the slope increase and for large $J$ is that the inviscid predictor of the momentum flux $\operatorname{Dr}_{G W P}$ becomes more and more accurate (see the gray dotted line in Figs. 4a and 4b).

If we now return to the total winds in Fig. 7 we also see that for large slope and large $J$, the winds along the upslope flank of the mountain become small compared to the downslope winds, an asymmetry that increases with both $S$ and $J$. More specifically, when $J=1$ (Fig. 7a) the flow contours the obstacle: the flow is upward on the upwind side and downward on the downwind side with little asymmetry in amplitudes, a behavior that is little affected by the increase in the slope in Fig. 7d. When $J$ increase and still for small slope in Figs. $7 \mathrm{~b}$ and $7 \mathrm{c}$ some upwind-downwind asymmetry starts to occur but stays limited: there is still substantial ascent on the upstream side of the obstacle. This ascent is actually not confined to the lower layers but extends up to at least twice the mountain slope. When the slope is larger (Figs. 7e and 7f), the upwind ascent is much smaller than the downwind descent. The downwind descent extends well along the downwind slope whereas along the upwind slope the total wind is very small. We call this situation a "nonseparated" blocking because it is produced by linear inflow dynamics.

To quantify the dependence on $S$ and $J$ more systematically in terms of upstream blocking and downslope winds, we plot in Fig. 8 the ratio between the wind amplitude along the downwind slope and the upwind slope of the ridge defined as

$$
\frac{\underbrace{\max }_{\bar{z}<2 \bar{h} / 3,0<\bar{x}<2} \sqrt{(\bar{z}+\bar{u})^{2}+\bar{w}^{2}}}{\underbrace{\max }_{\bar{z}<2 \bar{h} / 3,-2<\bar{x}<0} \sqrt{(\bar{z}+\bar{u})^{2}+\bar{w}^{2}}} .
$$

This ratio emphasizes more the downslope-upslope asymmetry than the criteria used in Lott (2016), where 


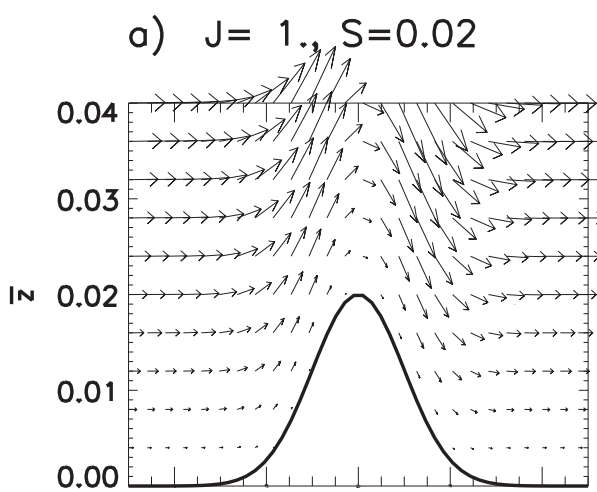

d) $J=1 ., S=0.15$

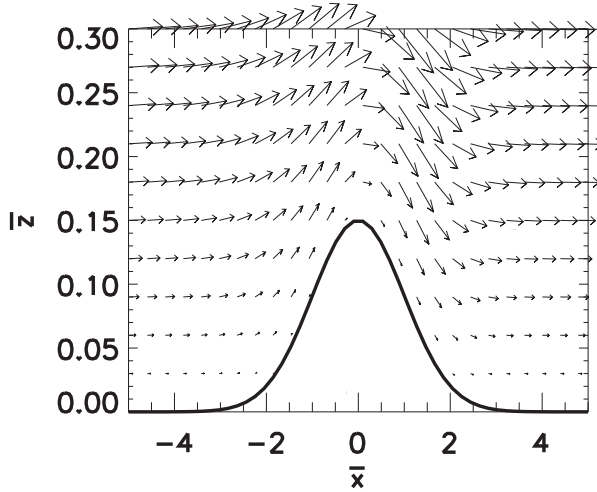

b) $\mathrm{J}=9 ., \mathrm{S}=0.02$

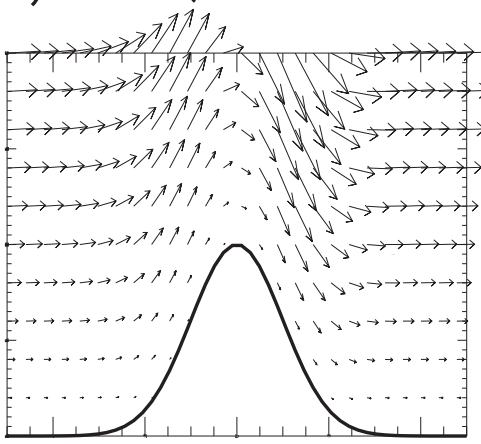

e) $J=9 ., S=0.15$

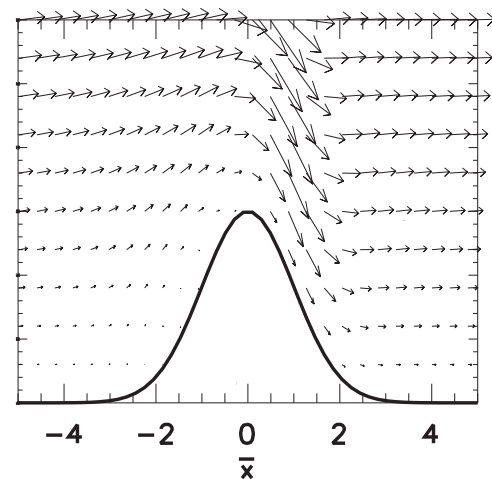

c) $\mathrm{J}=16 ., \mathrm{S}=0.02$

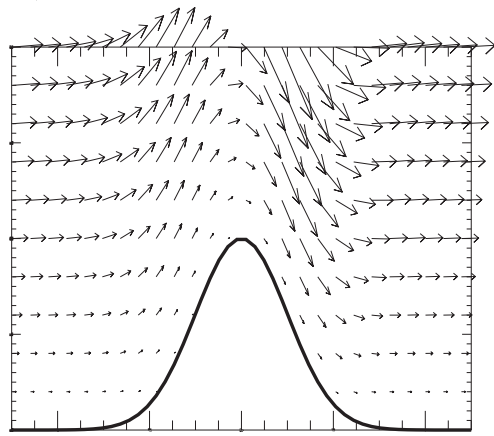

f) $J=16 ., S=0.15$

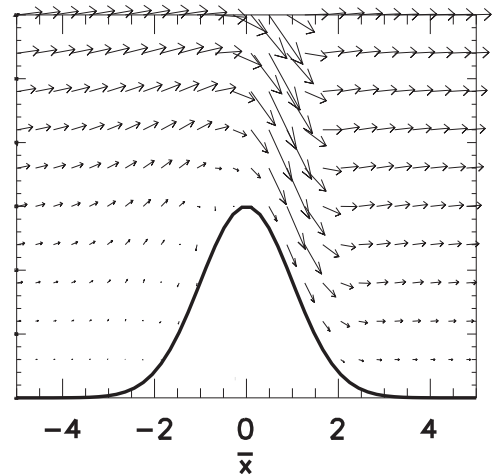

FIG. 7. Wind vectors around the hill and for different values of the Richardson number $J$ and of the slope $S$. Inner-layer depth $\delta(1)=0.1$.

only the downslope wind amplitude was measured in relation with the wind at the top of the hill. The reason is that here the wind at the top of the hill is null so this measure makes little sense. Here the ratio measures the upstream flow blocking as much as the downslope wind intensification and we see that it can easily reach values around 4 or 5 for slopes near the inner-layer depth $\delta(1)=0.1$ and when $J$ is sufficiently large. It always stays near 1 for small slopes and essentially increases with $J$ and $S$ as expected.

\section{Validation with a fully nonlinear model}

To validate our results we now use the ocean Massachusetts Institute of Technology General Circulation Model (MITgcm) (Marshall et al. 1997) and that solves the fully nonlinear Boussinesq hydrostatic equations on a cartesian mesh with a staggered Arakawa $\mathrm{C}$ grid. We set the shape of the topography to a Gaussian [Eq. (3)] and take $L=1 \mathrm{~km}$ and $H=150 \mathrm{~m}$, which yield $S=0.15$. Cells near the bottom are cut with the partial cells strategy (Adcroft et al. 1997) with $\mathrm{hFacmin}=0.1$ (if a fraction of the cell is less than hFacMin, then it is rounded to the nearer of 0 or hFacMin). The total domain horizontal size is $60 \mathrm{~km}$ with a stretched grid near the topography: the minimum and maximum grid size are 60 and $600 \mathrm{~m}$, respectively. We use a sponge layer at the lateral boundaries to relax the dynamic variables (momentum and temperature) to the prescribed upstream profiles (2). The relaxation time scale is $100 \mathrm{~s}$ in the innermost point of the sponge layer and $10 \mathrm{~s}$ in the outermost point of the sponge layer. We also

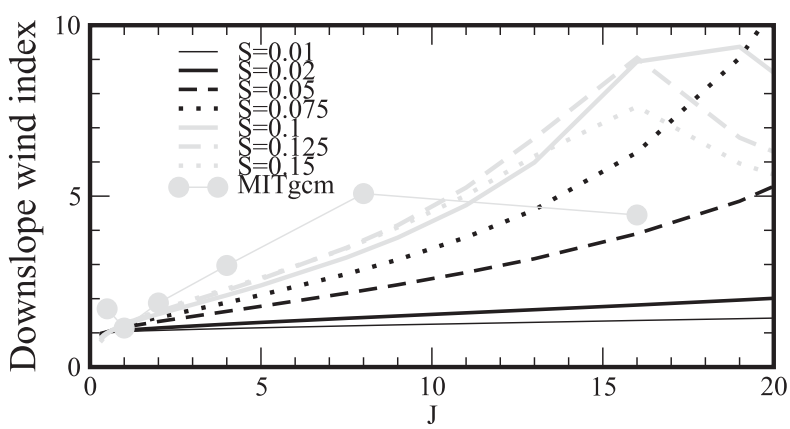

FIG. 8. Upstream blocking vs downslope windstorm index defined as the ratio between the maximum downslope wind amplitude and the maximum upslope wind amplitude. Gray dots are from the MITgcm with $S=0.15$. 
a)

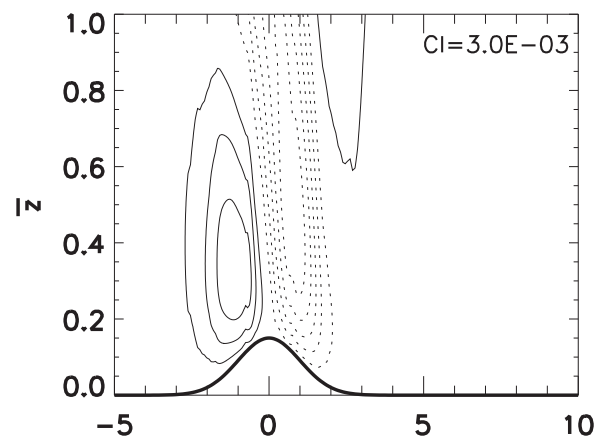

d) $\quad J=1, S=0.15$

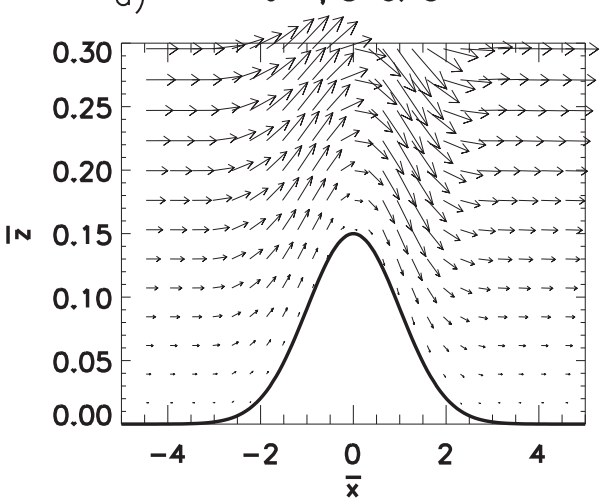

b)

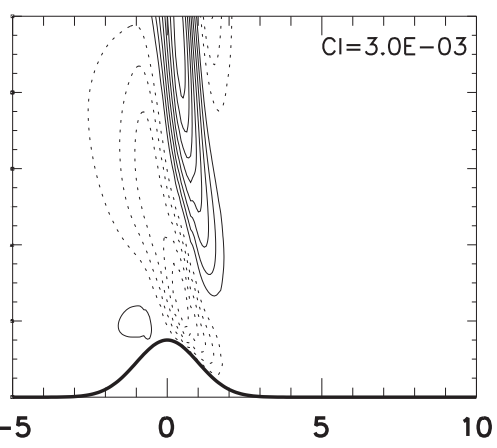

e)

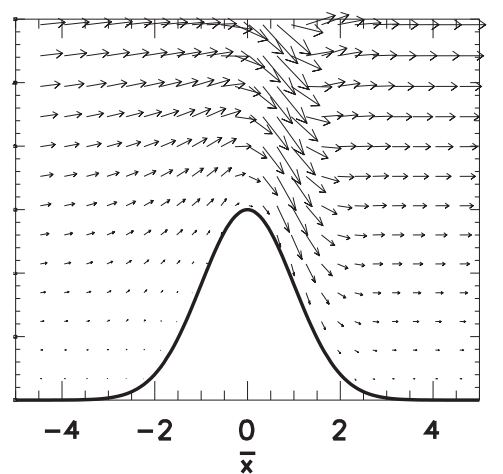

c) $\quad J=16, S=0.15$

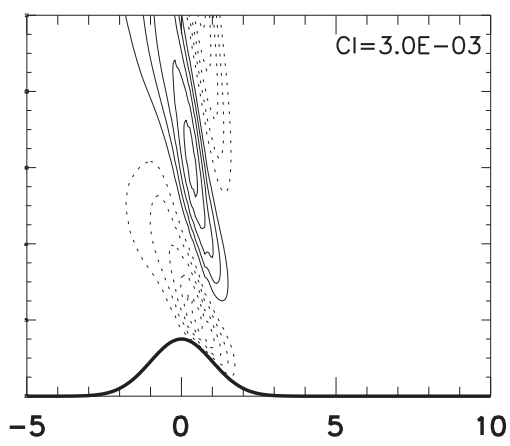

f)

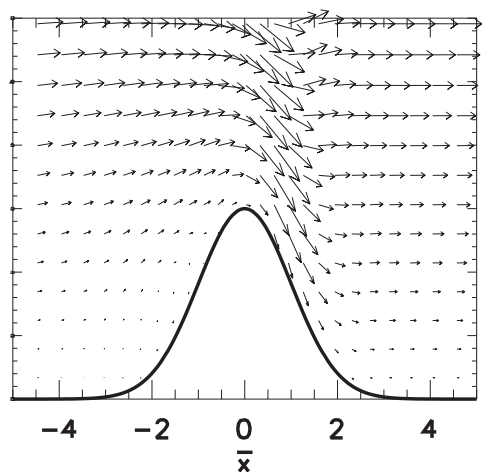

FIG. 9. (a)-(c) Vertical velocities from the MITgcm corresponding to the theory in Figs. 6d-f. (d)-(f) Wind vectors from the MITgcm corresponding to the theory in Figs. 7d-f.

use a stretched grid in the vertical with maximum resolution of $5.6 \mathrm{~m}$ at the topography and $415 \mathrm{~m}$ at the top of the domain. The total height of the domain is $50 \mathrm{~km}$, and we use a sponge layer above $10 \mathrm{~km}$ with a relaxation time scale that varies quadratically with a minimum time scale of $10 \mathrm{~s}$ at the uppermost grid point (and infinite relaxation time scale at $10 \mathrm{~km}$ ). We use a constant wind shear $\left(u_{0 z}=10^{-3} \mathrm{~s}^{-1}\right)$ and constant vertical temperature gradient. The temperature is related to the density via a linear equation of state and we adjust the vertical stratification $N^{2}$ to match the nondimensional values of $J$ : from $N^{2}=5 \times 10^{-7} \mathrm{~s}^{-2}(J=0.5)$ to $N^{2}=1.6 \times$ $10^{-5} \mathrm{~s}^{-2}(J=16)$. We use no-slip boundary conditions for momentum at the topography and we set the bottom temperature to $T=0^{\circ} \mathrm{C}$ (we modified the code to get a temperature flux at the boundary to ensure that the temperature at the topography is constant). The horizontal and vertical viscosities for momentum are set to $1 \mathrm{~m}^{2} \mathrm{~s}^{-1}$. The vertical and horizontal coefficients of diffusivity for temperature are set to $2 \mathrm{~m}^{2} \mathrm{~s}^{-1}$. We also added a horizontal biharmonic damping with a coefficient of $2 \times 10^{3} \mathrm{~m}^{4} \mathrm{~s}^{-1}$ for both the temperature and momentum in order to damp gridscale noise generated at the topography. The time step is $0.5 \mathrm{~s}$. The model is integrated forward in time until we reach a steady state (usually less than 1 day).

The results for the vertical velocity field in Figs. 9a-c, reproduce reasonably well the corresponding predictions from the theory in Figs. 6d-f, respectively. The horizontal scale and vertical variations are well reproduced, the amplitudes in the MITgcm are about $10 \%$ smaller near $\bar{z}=1$ than in the theory, a difference we attribute to numerical dissipations that are not easy to control. The results for the winds at low level in Figs. 9d-f are also consistent with those from the theory in Figs. 7d-f, respectively. The flow in the MITgcm presents the upstream/downstream symmetry predicted by theory when $J=1$ in Fig. 9a, and stronger downslope than upslope winds when $J=9$ and $J=16$. We conclude that there is a good agreement between the global indexes defined in the theoretical model and the fully nonlinear model (see for instance the comparison of the emitted wave fluxes in Figs. 3a and 3b, or of the downslope windstorm index in Fig. 8). The only noticeable difference is on the pressure drag in Fig. 2, the MITgcm predicts a larger drag than in the theory. We have tried to understand the causes of the differences, but find it difficult to correct the error. A reason is that the major 
differences between the theory and the MITgcm are essentially located near the surface (not shown), that is, at places where viscous stress equals the Reynolds stress and where the stepwise treatment of the lower boundary can produce gridscale irregularities on these fields. As such irregularities are likely to be damped out by dissipation as we move away from the surface, we can speculate that these low-level differences on the velocity shears and pressure are not significant in the context of the interactions between the waves and the large-scale flow at upper levels.

\section{Conclusions}

In dynamical meteorology and oceanography, solutions with constant viscosity have always been a starting points to understand phenomena that involve the interaction between the surface and the boundary layer. Examples are numerous, from the Ekman (1905) solutions systematically given in textbooks, the Prandtl (1952) model for katabatic winds, the inclusion of a boundary layer in the Miles theory for the generation of oceanic waves (Benjamin 1959), or in theories of sand ripples and dunes formation (Engelund 1970) (see also Fowler 2001). In waves and dune theories, the fact that the near-surface wind profiles play a crucial role in the dynamics was early recognized (Miles 1957; Benjamin 1959), and a first difficulty consisted in solving the fourth-order Orr-Sommerfeld equation and to introduce a corrugated bottom at the surface (Fowler 2001). A difficulty arises if one wishes to introduce stratification: the equation to solve becomes of the sixth order [see Eq. (17)]. This difficulty plus the facts that a constant eddy viscosity is a crude approximations of the turbulence in actual boundary layers, are two reasons why the viscous problem is not often treated in the stratified case. When it is, the techniques used are extremely involved [see, for instance, the introduction of "triple decks" in Sykes (1978)], and does not permit to derive uniform approximations of the solutions over the entire domain. As this last remark also holds for more sophisticated eddy viscosity closure, it is fair to say that theories failed so far in predicting the vertical profiles of the wave Reynolds stress, a quantity that is central in mountain meteorology. For these reasons but also because more and more papers in mountain meteorology call for a better understanding of the interaction between boundary layers and mountain waves, we found useful to solve the viscous mountain wave problem theoretically, and verify the theory with a fully nonlinear model (here the MITgcm). Note that in the context of stratified oceanic boundary layers over corrugated and tilted slopes, a recent paper by Passaggia et al. (2014) shares the same concern.

Once given this context, what are the messages that could be useful in a more realistic context? The first is probably that pressure drag and wave Reynolds stress are well predicted by inviscid theory and if we take for the incident flow, its value averaged over the innerlayer depth. This depth has a definition that can be generalized, at least conceptually. For instance, if the boundary layer scheme uses first-order closure with vertical diffusion coefficients, the coefficients and tendencies can be linearized around the large-scale resolved state. If we consider a small perturbation of given horizontal scale, the inner-layer depth of interest is that where advection by the resolved wind equals the disturbance in boundary layer tendency. These predictions of the drag and waves Reynolds stress remain valid until the mountain height equals the inner-layer scale. Our theory does not go beyond that height. For higher mountains we should probably average the incident flow over the mountain height to obtain realistic predictions. Actually, this is what we find with our model when imposing free slip boundary condition in $\bar{z}=\bar{h}$, that is, in an inviscid approximation where the boundary layer depth is drastically reduced (not shown).

For large values of the stratification, we also find that a good fraction of the stress is dissipated near the top of the inner layer, simply because the waves have shorter vertical wavelength and are more dissipated there. This effect is mitigated when the top of the hill is near the top of the inner-layer scale again, but suggests that a good fraction of the mountain wave drag should be given back to the flow near the top of the inner layer. Another interesting result concerns the source of the mountain wave stress. When the mountain is well inside the inner layer, the wave stress is in good part extracted from the inner layer itself rather than from the solid earth as in the inviscid case. When the mountain slope approaches the inner-layer depth this result is less applicable and a good part of the pseudo momentum flux is directed toward the surface as in the inviscid case (Durran 1995; Lott 1998).

Our results could also be used to provide alternative views concerning the dynamics of upstream blocking and downslope winds. They occur through a nearsurface critical-level dynamics and without upperlevel wave breaking (remember that our theory is linear inside the flow) providing that the flow is stable $J>1$, and that the mountain slope is near the innerlayer scale. This confirms the results in Lott (2016) and Damiens et al. (2018) who predicted these behaviors using simpler theories and using simulations with 
WRF including more sophisticated boundary layers. Another important result concerns the structure of the inner layer itself: the downslope winds penetrate well into it, as shown for instance in Fig. 7 when $J=9$ or $J=16$.

Last, for all the results presented here, we have neglected that the mountain gravity waves necessarily return to the surface in the constant shear case: they are all trapped, and this effect should be taken into account to give a more realistic treatment of the constant shear case. To take this into account within our theoretical framework we need to reject the hydrostatic approximation and we have to treat the inviscid solution in terms of Hankel functions (Keller 1994), a solution we will describe in a future paper. Note that such subsequent development will also allow us to treat the nonstratified situation and describe the transition from the neutral to the stratified case. Here we wanted to treat the hydrostatic case first because an extremely rich dynamics already occur at the low level and we do not need to attribute this dynamics to the fact that all the harmonics are trapped.

In this paper also, the background shear flow is constant, which corresponds to a boundary layer flow of infinite depth. Hence, even though we insist on using the terminology that the dynamics introduces an "inner" layer scale, it has to be clearly distinguished from the plausible presence of a "boundary layer," where the incident wind present large curvature. Again, we can treat such problem with our formalism by imposing background flow with nonzero curvature, a situation that can introduced trapped lee waves in the nonhydrostatic case (Soufflet et al. 2019).

Acknowledgments. This work was supported by the Laboratoire de Recherche Conventionné "Yves Rocard," a collaborative unit between CEA and Ecole Normale Supérieure.

\section{APPENDIX A}

\section{Preconditioning of the Viscous Solution}

To evaluate $\tilde{\mathbf{w}}_{2}, \tilde{\mathbf{w}}_{3}$, and $\tilde{\mathbf{w}}_{4}$ we proceed as in Lott (2007), take the asymptotic forms in Eq. (18) when $\tilde{z}>5$ and integrate down to $\tilde{z}=0$ with a RungeKutta algorithm. Nevertheless these solutions are illconditioned when it comes to the inversion of the boundary condition, essentially because $\tilde{\mathbf{w}}_{3}$ and $\tilde{\mathbf{w}}_{4}$ vary exponentially with altitude [see Eq. (18)]. To circumvent this difficulty, rather than $\tilde{\mathbf{w}}_{2}, \tilde{\mathbf{w}}_{3}$, and $\tilde{\mathbf{w}}_{4}$ we have used 3 solutions $\tilde{\mathbf{w}}_{a}, \tilde{\mathbf{w}}_{b}$, and $\tilde{\mathbf{w}}_{c}$, whose asymptotic behavior for $\tilde{z} \rightarrow \infty$ all match the inviscid solution $\overline{\mathbf{w}}_{I}$ but do not grow exponentially fast when $\tilde{z} \rightarrow 0$ :

$$
\begin{aligned}
& \tilde{\mathbf{w}}_{a}(\tilde{z})=\tilde{\mathbf{w}}_{2}(\tilde{z})+\tilde{a}_{3} \tilde{\mathbf{w}}_{3}(\tilde{z})+\tilde{a}_{4} \tilde{\mathbf{w}}_{4}(\tilde{z}), \\
& \tilde{\mathbf{w}}_{b}(\tilde{z})=\tilde{\mathbf{w}}_{2}(\tilde{z})+\tilde{b}_{3} \tilde{\mathbf{w}}_{3}(\tilde{z})+\tilde{b}_{4} \tilde{\mathbf{w}}_{4}(\tilde{z}), \\
& \tilde{\mathbf{w}}_{c}(\tilde{z})=\tilde{\mathbf{w}}_{2}(\tilde{z})+\tilde{c}_{3}(\bar{k})(\tilde{z})+\tilde{c}_{4} \tilde{\mathbf{w}}_{4}(\tilde{z}) .
\end{aligned}
$$

The three pairs $\left(\tilde{a}_{3}, \tilde{a}_{4}\right),\left(\tilde{b}_{3}, \tilde{b}_{4}\right)$, and $\left(\tilde{c}_{3}, \tilde{c}_{4}\right)$ are then chosen so that $\left[\partial_{\tilde{z}} \tilde{\mathbf{u}}_{a}(0), \partial_{\tilde{z}} \tilde{\mathbf{b}}_{a}(0)\right]=(0,0),\left[\partial_{\tilde{z}} \tilde{\mathbf{u}}_{b}(0)\right.$, $\left.\tilde{\mathbf{p}}_{b}(0)\right]=(0,0)$, and $\left[\partial_{\tilde{z}} \tilde{\mathbf{b}}_{c}(0), \tilde{\mathbf{p}}_{c}(0)\right]=(0,0)$, respectively. These three solutions are shown in Fig. A1 for $J=1$ and $\operatorname{Pr}=0.5$, they show moderate variations with inner altitude $\tilde{z}$, the exponential behavior of $\tilde{\mathbf{w}}_{3}$ and $\tilde{\mathbf{w}}_{4}$ has clearly been mitigated by adopting finite amplitudes values for the variables and their derivatives at the surface. The boundary condition is then satisfied by writing

$$
\begin{aligned}
\bar{w}(\bar{x}, \bar{h}) \approx & \int_{-\infty}^{+\infty} \bar{k} \bar{\delta}(\bar{k})\left[f_{a}(\bar{k}) \tilde{\mathbf{w}}_{a}(\tilde{h})+f_{b}(\bar{k}) \tilde{\mathbf{w}}_{b}(\tilde{h})\right. \\
& \left.+f_{c}(\bar{k}) \tilde{\mathbf{w}}_{c}(\tilde{h})\right] e^{i \bar{k} \bar{x}} d \bar{k}=0 \\
\bar{u}(\bar{x}, \bar{h}) \approx & \int_{-\infty}^{+\infty}\left[f_{a}(\bar{k}) \tilde{\mathbf{u}}_{a}(\tilde{h})+f_{b}(\bar{k}) \tilde{\mathbf{u}}_{b}(\tilde{h})\right. \\
& \left.+f_{c}(\bar{k}) \tilde{\mathbf{u}}_{c}(\tilde{h})\right] e^{i \bar{k} \bar{x}} d \bar{k}=-\bar{h}(\bar{x}) \\
\bar{b}(\bar{x}, \bar{h}) \approx & \int_{-\infty}^{+\infty}\left[f_{a}(\bar{k}) \tilde{\mathbf{b}}_{a}(\tilde{h})+f_{b}(\bar{k}) \tilde{\mathbf{b}}_{b}(\tilde{h})\right. \\
& \left.+f_{c}(\bar{k}) \tilde{\mathbf{b}}_{c}(\tilde{h})\right] e^{i \bar{k} \bar{x}} d \bar{k}=-J \bar{h}(\bar{x})
\end{aligned}
$$

where $\tilde{h}(\bar{x}, \bar{k})=\bar{h}[\bar{x} / \bar{\delta}(\bar{k})]$. Once discretized in the horizontal and spectral domain, the set of Eqs. (20) corresponds to three linear equations involving nine matrices [for instance one of the matrices has for components $\left.\overline{k_{j}} \bar{\delta}\left(\bar{k}_{j}\right) \tilde{\mathbf{w}}_{a}\left(\tilde{h}_{i j}\right) e^{i \bar{k}_{j} \bar{x}_{i}} d \bar{k}\right]$ and three unknown vectors [with components $f_{a}\left(\bar{k}_{j}\right), f_{b}\left(\bar{k}_{j}\right)$, and $\left.f_{c}\left(\bar{k}_{j}\right)\right]$ that can be inverted with conventional matrix inversion routines.

Still in this formalism, the uniform approximation of $\overline{\mathbf{w}}$ in Eq. (19) writes

$$
\begin{aligned}
\overline{\mathbf{w}}(\bar{k}, \bar{z})= & \bar{k} \bar{\delta}(k)\left\{f_{a}(\bar{k}) \tilde{\mathbf{w}}_{a}[\bar{k}, \bar{z} / \bar{\delta}(\bar{k})]\right. \\
& \left.+f_{b}(\bar{k}) \tilde{\mathbf{w}}_{b}[\bar{k}, \bar{z} / \bar{\delta}(\bar{k})]+f_{c}(\bar{k}) \tilde{\mathbf{w}}_{c}[\bar{k}, \bar{z} / \bar{\delta}(\bar{k})]\right\},
\end{aligned}
$$

again with similar expression for $\overline{\mathbf{u}}$ and $\overline{\mathbf{b}}$. 

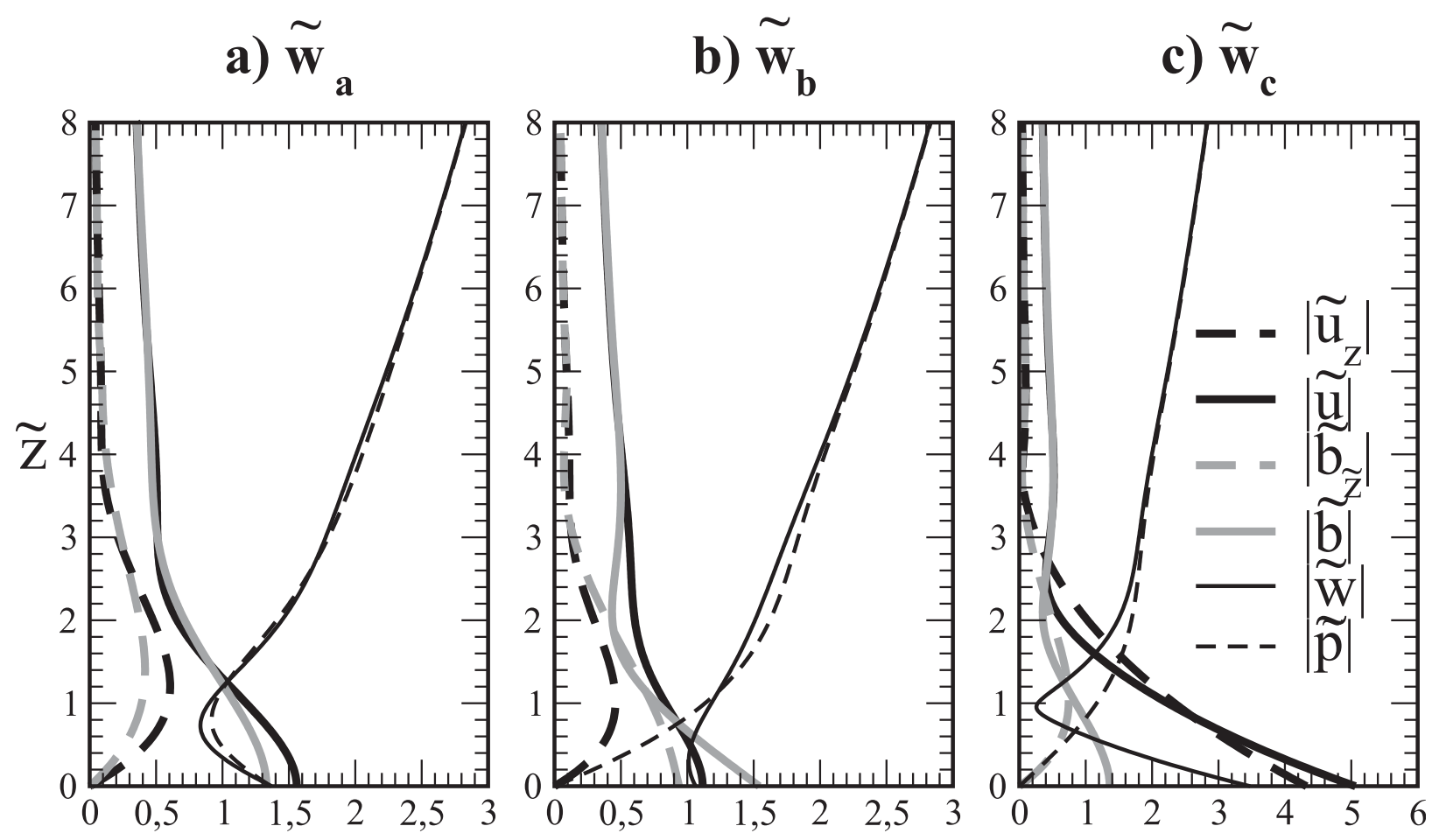

FIG. A1. Uniform solutions used to invert boundary conditions and to evaluate the wave fields over the entire domain. $J=1$ and Pr $=0.5$. All the solutions are expressed using inner variables.

\section{APPENDIX B}

\section{Numerical Resolution}

To solve numerically our problem we always take a domain of length $\bar{X}=100$ spanned by $N=1024$ points, which corresponds to a spectral resolution around $d \bar{k} \approx 0.01$ and a spatial resolution around $d \bar{x} \approx 0.1$. In the vertical we take grids of maximum depth $\bar{Z}=3$ and smoothly varying vertical resolution. The variable resolution is such that for $\bar{z}>10 S$ the grid spacing $d z \approx$ 0.03 whereas near around and below the mountain top $d z \approx S / 10$. We will then systematically vary the other two nondimensional parameters of the problem $S$ and $J$.

Concerning the variations in slope $S$, we have to assume that the mountain is well in the inner layer, a condition that needs to be satisfied for each harmonics. Although this pauses a theoretical problem since in the infinite Fourier integrals $\bar{k}$ can become extremely large [and $\bar{\delta}(\bar{k})$ very small] it can be handled numerically once fixed the horizontal scale of the domain over which Fourier series approximate Fourier transform and once fixed the number of horizontal grid points. More specifically, if $\bar{k}_{\max }=N \pi / X$, the condition that the associated inner-layer depth is larger than the mountain top is
$S / \bar{\delta}\left(\bar{k}_{\max }\right) \approx 1$ or less. Nevertheless, and for moderately large domain length $\bar{X}$ it happens that it is sufficient to satisfy this condition for the dominant wavenumbers, that is, to satisfy $S / \bar{\delta}(1) \ngtr 1$. This guaranties that the dominant harmonics forced by the obstacle are still well viscous near the mountain top. In this case, numerical convergence was found up to around $S \approx 0.15$.

\section{REFERENCES}

Adcroft, A., C. Hill, and J. Marshall, 1997: Representation of topography by shaved cells in a height coordinate ocean model. Mon. Wea. Rev., 125, 2293-2315, https://doi.org/10.1175/15200493(1997)125<2293:ROTBSC $>2.0$. CO;2.

Ayotte, K., 2008: Computational modelling for wind energy assessment. J. Wind Eng. Ind. Aerodyn., 96, 1571-1590, https:// doi.org/10.1016/j.jweia.2008.02.002.

Baldwin, P., and P. H. Roberts, 1970: The critical layer in stratified shear flow. Mathematika, 17, 102-119, https://doi.org/10.1112/ S0025579300002783.

Belcher, S. E., and N. Wood, 1996: Form and wave drag due to stably stratified turbulent flow over low ridges. Quart. J. Roy. Meteor. Soc., 122, 863-902, https://doi.org/10.1002/ qj.49712253205.

Beljaars, A. C. M., J. Walmsley, and P. Taylor, 1987: A mixed spectral finite-difference model for neutrally stratified boundary-layer flow over roughness changes and topography. Bound.-Layer Meteor., 38, 273-303, https://doi.org/ 10.1007/BF00122448. 
_ A. R. Brown, and N. Wood, 2004: A new parametrization of turbulent orographic form drag. Quart. J. Roy. Meteor. Soc., 130, 1327-1347, https://doi.org/10.1256/qj.03.73.

Benjamin, T. B., 1959: Shearing flow over a wavy boundary. J. Fluid Mech., 6, 161-205, https://doi.org/10.1017/S0022112059000568.

Booker, J. R., and F. P. Bretherton, 1967: The critical layer for internal gravity waves in a shear flow. J. Fluid Mech., 27, 513539, https://doi.org/10.1017/S0022112067000515.

Charru, F., B. Andreotti, and P. Claudin, 2013: Sand ripples and dunes. Annu. Rev. Fluid Mech., 45, 469-493, https://doi.org/ 10.1146/annurev-fluid-011212-140806.

Damiens, F., F. Lott, C. Millet, and R. Plougonven, 2018: An adiabatic foehn mechanism. Quart. J. Roy. Meteor. Soc., 144, 1369-1381, https://doi.org/10.1002/qj.3272.

Doyle, J. D., and Coauthors, 2011: An intercomparison of T-REX mountain-wave simulations and implications for mesoscale predictability. Mon. Wea. Rev., 139, 2811-2831, https://doi.org/ 10.1175/MWR-D-10-05042.1.

Durran, D. R., 1990: Mountain waves and downslope winds. Atmospheric Processes over Complex Terrain, Meteor. Monogr., No. 23, Amer. Meteor. Soc., 59-83.

- 1995: Pseudomomentum diagnostics for two-dimensional stratified compressible flow. J. Atmos. Sci., 52, 3997-4009, https:// doi.org/10.1175/1520-0469(1995)052<3997:PDFTDS>2.0.CO;2.

Ekman, V. W., 1905: On the influence of the Earth's rotation on ocean-currents. Arch. Math. Astron. Phys., 2, 1-52.

Eliassen, A., and E. Palm, 1961: On the transfer of energy in stationary mountain waves. Geofys. Publ., 22, 1-23.

Engelund, F., 1970: Instability of erodible beds. J. Fluid Mech., 42, 225-244, https://doi.org/10.1017/S0022112070001210.

Fowler, A. C., 2001: Dunes and drumlins. Geomorphological Fluid Mechanics, N. J. Balmforth and A. Provenzale, Eds., Springer, 430-454.

Hazel, P., 1967: The effect of viscosity and heat conduction on internal gravity waves at a critical level. J. Fluid Mech., 30, 775783, https://doi.org/10.1017/S0022112067001752.

Hunt, J. C. R., S. Leibovich, and K. J. Richards, 1988: Turbulent shear flows over low hills. Quart. J. Roy. Meteor. Soc., 114, 1435-1470, https://doi.org/10.1002/qj.49711448405.

Jackson, P. S., and J. C. R. Hunt, 1975: Turbulent wind flow over low hill. Quart. J. Roy. Meteor. Soc., 101, 929-955, https:// doi.org/10.1002/qj.49710143015.

Jiang, Q., J. D. Doyle, and R. B. Smith, 2006: Interaction between trapped waves and boundary layers. J. Atmos. Sci., 63, 617633, https://doi.org/10.1175/JAS3640.1.

Keller, T. L., 1994: Implications of the hydrostatic assumption on atmospheric gravity waves. J. Atmos. Sci., 51, 1915-1929, https:// doi.org/10.1175/1520-0469(1994)051<1915:IOTHAO>2.0.CO;2.

Lott, F., 1998: Linear mountain drag and averaged pseudo-momentum flux profiles in the presence of trapped lee waves. Tellus, $\mathbf{5 0 A}, 12$ 25, https://doi.org/10.3402/tellusa.v50i1.14509.

_ 2007: The reflection of a stationary gravity wave by a viscous boundary layer. J. Atmos. Sci., 64, 3363-3371, https://doi.org/ 10.1175/JAS4020.1.

__ 2016: A new theory for downslope windstorms and trapped lee waves. J. Atmos. Sci., 73, 3585-3597, https://doi.org/ 10.1175/JAS-D-15-0342.1.

_ rametrization: Its formulation and testing. Quart. J. Roy. Meteor. Soc., 123, 101-127, https://doi.org/10.1002/qj.49712353704.

Marshall, J., A. Adcroft, C. Hill, L. Perelman, and C. Heisey, 1997: A finite-volume, incompressible Navier Stokes model for studies of the ocean on parallel computers. J. Geophys. Res., 102, 5753-5766, https://doi.org/10.1029/96JC02775.

Miles, J. W., 1957: On the generation of surface waves by shear flows. J. Fluid Mech., 3, 185-204, https://doi.org/10.1017/ S0022112057000567.

Ólafsson, H., and P. Bougeault, 1997: The effect of rotation and surface friction on orographic drag. J. Atmos. Sci., 54, 193-210, https:// doi.org/10.1175/1520-0469(1997)054<0193:TEORAS>2.0.CO;2.

Passaggia, P.-Y., P. Meunier, and S. Le Dizes, 2014: Response of a stratified boundary layer on a tilted wall to surface undulations. J. Fluid Mech., 751, 663-684, https://doi.org/10.1017/ jfm.2014.296.

Pithan, F., T. G. Shepherd, G. Zappa, and I. Sandu, 2016: Climate model biases in jet streams, blocking and storm tracks resulting from missing orographic drag. Geophys. Res. Lett., 43, 7231-7240, https://doi.org/10.1002/2016GL069551.

Prandtl, L., 1952: Essentials of Fluid Dynamics. Blackie, 452 pp.

Richard, E., P. Mascart, and E. C. Nickerson, 1989: The role of surface friction in downslope windstorms. J. Appl. Meteor., 28, 241-251, https://doi.org/10.1175/1520-0450(1989)028<0241: TROSFI $>2.0 . \mathrm{CO} ; 2$.

Sandu, I., P. Bechtold, A. Beljaars, A. Bozzo, F. Pithan, T. Shepherd, and A. Zadra, 2015: Impacts of parameterized orographic drag on the Northern Hemisphere winter circulation. J. Adv. Model. Earth Syst., 8, 196-211, https://doi.org/10.1002/2015MS000564.

Scinocca, J., and T. Shepherd, 1992: Nonlinear wave-activity conservation laws and Hamiltonian structure for the two-dimensional anelastic equations. J. Atmos. Sci., 49, 5-28, https://doi.org/ 10.1175/1520-0469(1992)049<0005:NWACLA > 2.0.CO;2.

Sheridan, P. F., V. Horlacherxi, G. G. Rooney, P. Hignett, S. D. Mobbs, and S. Vosper, 2007: Influence of lee waves on the near surface flow downwind of the Pennines. Quart. J. Roy. Meteor. Soc., 133, 1353-1369, https://doi.org/10.1002/QJ.110.

— S. Vosper, and P. Brown, 2017: Mountain waves in high resolution forecast models: Automated diagnostics of wave severity and impact on surface winds. Atmosphere, 8, 24, https://doi.org/10.3390/atmos8010024.

Smith, F. T., 1973: Laminar flow over a small hump on a flat plate. J. Fluid Mech., 57, 803-824, https://doi.org/10.1017/ S002211207300203X.

Smith, R. B., S. Skubis, J. D. Doyle, A. S. Broad, C. Kiemle, and H. Volkert, 2002: Mountain waves over Mont Blanc: Influence of a stagnant boundary layer. J. Atmos. Sci., 59, 2073-2092, https:// doi.org/10.1175/1520-0469(2002)059<2073:MWOMBI>2.0.CO;2.

, Q. Jiang, and J. D. Doyle, 2006: A theory of gravity wave absorption by a boundary layer. J. Atmos. Sci., 63, 774-781, https://doi.org/10.1175/JAS3631.1.

Soufflet, C., F. Lott, and F. Damiens, 2019: Trapped mountain waves with critical level just below the surface. Quart. J. Roy. Meteor. Soc., 145, 1503-1514, https://doi.org/10.1002/qj.3507.

Sykes, R., 1978: Stratification effects in boundary layer flow over hills. Proc. Roy. Soc. London, 361A, 225-243, https://doi.org/ 10.1098/RSPA.1978.0100.

Van Duin, C. A., and H. Kelder, 1986: Internal gravity waves in shear flows at large Reynolds number. J. Fluid Mech., 169, 293-306, https://doi.org/10.1017/S0022112086000642.

Wood, N., and P. Mason, 1993: The pressure force induced by neutral, turbulent flow over hills. Quart. J. Roy. Meteor. Soc., 119, 1233-1267, https://doi.org/10.1002/qj.49711951402.

A. R. Brown, and F. E. Hewer, 2001: Parameterizing the effects of orography on the boundary layer: An alternative to effective roughness lengths. Quart. J. Roy. Meteor. Soc., 127, 759-777, https://doi.org/10.1256/SMSQJ.57302. 